\title{
Collective Memory, Cultural Transmission and Investments
}

\author{
June 23, 2007
}

\begin{abstract}
I study the intergenerational transmission of collective memory as a mechanism for cultural transmission, in the presence of social externalities associated with individual cultural investment decisions (learning and internalizing cultural norms and values). The younger generation's investment decisions are based on beliefs about the quality of existing institutions, norms and values; these beliefs are influenced by the information (memory) received from the older generation. I find that in culturally homogeneous societies it can be optimal to suppress "negative" memories while emphasizing and elaborating "positive" ones. However, the ability to bias collective memory in this way has important costs: it may generate cultural over-optimism and over-investment in some cases, and the reverse, cultural pessimism and under-investment, in other cases. The scope for welfare-enhancing manipulation of collective memory is reduced, moreover, in culturally heterogeneous societies. My findings are consistent with the empirical evidence on the evolution of collective memory in a variety of countries.
\end{abstract}

JEL classification numbers: D82, D83, Z13.

Keywords: memory, identity, cultural transmission, externalities, history, multi-cultural. 


\section{Introduction}

Memory of the past plays a crucial role in the transmission of cultural and national identity. In every society and every country, the collective memory ${ }^{1}$ transmitted to the young by the older generation, through a variety of channels (e.g. school textbooks, the media, monuments and commemorative rituals), influences their perception of their cultural identity and values, and their willingness to invest in them - with major economic as well as political and social consequences. This naturally raises two questions. First, what would be the socially optimal transmission of collective memory to the young? Is it the case, as intuition might suggest, that the older generation should strive to be as unbiased, accurate and comprehensive as possible in communicating its collective memory to the younger generation? If not, why and how should it depart from this objective? Second, is collective memory transmission in practice likely to differ from what would be socially optimal, and if so how? These questions are at the heart of many current debates, including those over education policy (e.g. how should history be taught? how much control should the state have over school textbooks and curriculum? $)^{2}$, over the media (e.g. the role of the CPB in the US or the BBC in the UK) ${ }^{3}$, and over cultural integration (e.g. centralized versus decentralized institutions in the European Union, legislation affecting freedom of speech in the UK $)^{4}$.

The present paper investigates the socially optimal transmission of collective memory to the young and the biases that are likely to arise in practice, in the light of the available empirical evidence on collective memory. Building on insights from the recent literature in economics and psychology, which has highlighted the potential benefits and costs of selective memory at the individual level ${ }^{5}$, I

\footnotetext{
${ }^{1}$ The term "collective memory" typically refers to a nation's or group's representations and associated interpretations of the past, communicated through narratives (e.g. textbooks, novels, films, newspapers, television) and symbols (e.g. monuments, art, commemorative rituals). These narratives and symbols convey information about the nation's or group's values and norms. See section 2 for a fuller discussion and examples.

${ }^{2}$ These questions have attracted considerable interest following the recent controversy over Japanese history textbooks (on this see, for example, http://news.bbc.co.uk/1/hi/world/ asia-pacific/4416593.stm;http://en.wikipedia.org/wiki/

Japanese_history_textbook_controversies).

${ }^{3}$ See for example Paul Fahri (April 22, 2005; June 10, 2005; November 4, 2005), The Washington Post.

${ }^{4}$ See for example Ben Russell (October 26, 2005), The Independent.

${ }^{5}$ In the economics literature, Bénabou and Tirole (2002) have analyzed the implications of selective memory for motivation when individuals are time-inconsistent. Compte and Postle-
} 
examine the costs and benefits associated with selective collective memory. The first part of the paper explores a simple intuition. In any society, when individuals invest in learning and internalizing that society's cultural norms and values, they exert significant externalities on other members of society. In very many cases, these will be positive externalities, as individuals adopt norms of cooperation, tolerance, fairness, honesty, and so on. Individuals will only be willing to invest in this way if they are sufficiently confident that the future returns (psychological as well as practical) will justify incurring current costs. Their investment decisions will therefore depend on their beliefs about the key determinants of these returns, including the quality of the shared values, norms and institutions, i.e. the culture, of the society (nation, community) to which they belong.

For example, making the necessary effort to learn self-discipline and how to work effectively requires a belief that hard work "pays" ${ }^{6}$; investing time and resources in ways that directly benefit others often requires a belief in norms of reciprocity ${ }^{7}$; investments by immigrants in learning and internalizing the host country's culture require a belief that this new identity will bring sufficient rewards; and investing in learning and adopting as one's own norms of fairness and tolerance requires a belief that others can, in general, be trusted to follow the same norms. At the time when the young have to take many of these investment decisions, their beliefs are strongly influenced by the information they receive from the older generation (e.g. at school, through the media) ${ }^{8}$. This suggests that the

waite (2004) have shown that when confidence has a positive effect on performance, biases in information processing, including selective memory, can enhance individual welfare. Both papers discuss extensively the related psychology literature.

${ }^{6}$ On the importance of such beliefs for investments, see Bénabou and Tirole (2006) and the evidence cited therein. One of the examples they give nicely captures the link: "My mom always told me that hard work, loyalty and respect for others will bring me success, wrote J.K., who was let go from Credit Suisse in late October. That's why I came back to CSFB after business school... and did all that other stuff. Apparently, it doesn't always work that way" [New York Times, December 1, 2002].

${ }^{7}$ See Falk and Zehnder (2006) for recent evidence on this from a field experiment conducted in Zurich. About 1,000 inhabitants of the city took part in a sequential trust game, in which first movers could condition their investments on the residential districts of second movers. The results show that first movers were able to correctly anticipate different levels of trustworthiness in different districts, and discriminated accordingly (i.e., sending more money to participants in districts where they were more likely to get more money back). Thus beliefs about differences in norms of reciprocity across districts strongly influenced investment behavior.

${ }^{8}$ This is obviously true for children and adolescents, but it is also largely the case for young adults, since searching individually for information is costly and the value of information is often very difficult to predict. Thus readily available information, selected and transmitted by the 
selection and presentation of information to the young could be used beneficially to "internalize" the externalities associated with individual investment decisions.

To investigate this possibility and its implications, I begin by studying the benchmark case of a culturally homogeneous society. I formalize this by letting an informed "principal" represent the older generation, and two identical "agents" represent the younger generation. The principal selects the information to be transmitted to the agents; each agent, in the light of the information he receives, will then make an investment decision that exerts an externality on the other agent. This is the simplest framework I can use to study the benchmark case; it will be modified and extended in the second part of the paper to allow for cultural heterogeneity. To examine the socially optimal transmission of memory in this setting, I assume that the principal chooses the information to be transmitted to the agents so as to maximize their welfare. When the externality between the agents is sufficiently large and positive, I find that it can indeed be optimal to select and present information to the young so as to foster optimism about the value of the existing culture, thereby inducing greater investments than would be possible with full and accurate transmission of information.

In practice, this may be achieved in several ways: first, by neglecting to communicate information that represents "bad news" about the value of the existing culture. Second, by presenting and interpreting the information in such a way as to effectively suppress the underlying bad signal. Third, by emphasizing and focusing attention on information that represents "good news", thereby distracting attention from any bad signal. There is a large body of empirical evidence on collective memory, discussed in section 2 , that provides numerous examples of the occurrence of all three, in many different situations, and many countries ${ }^{9}$.

Whilst the analysis in this first part of the paper identifies a potential benefit from the selective emphasis, suppression and elaboration of the memory transmitted to the young, it also highlights some of the potential costs. First, when the underlying signal is bad, suppression may give rise to cultural over-optimism and hence over-investment. One possible interpretation of cultural over-investment is

older generation, tends to be particularly salient and has a substantial impact on beliefs.

${ }^{9}$ There is clearly an analogy here with some of the ways in which "bad signals" are suppressed at the individual level. Psychologists have found evidence of self-serving attributions (people tend to attribute their successes more easily to their ability, their failures to the difficulty of the task or bad luck). They have also found evidence that individuals examine more carefully "negative" information, in order to find flaws in it, or develop alternative explanations (i.e. explanations that largely suppress the underlying bad signal). For a review and discussion of this evidence see Dunning (2001). 
the tendency to invest so much in learning and internalizing the existing cultural values of the society that insufficient attention is given to critical questioning, or comparing and learning from other societies' cultures. When the underlying signal is good, the opposite problem may arise: to the extent that the young are aware of the likely bias towards suppressing bad signals, they will distrust the "positive" information transmitted by the older generation, and they will be unduly pessimistic about the value of their culture, leading to under-investment. This second cost is only present if the young are sufficiently "Bayesian", as discussed in section 4, while the first cost can arise even when the young are "naive".

The results just described were obtained by focusing on the maximization of the younger generation's welfare. The analysis can easily be generalized to allow also for a direct impact on the well-being of the older generation: For example, the older generation may incur costs associated with investments by the young (e.g. as parents, teachers, mentors and role models); on the other hand, it may also derive psychological and practical benefits from successfully transmitting its values and norms to the young. If the net direct benefits to the older generation are strictly positive (negative), the net gains from suppressing bad signals will be correspondingly higher (lower). In practice, the older generation may not fully internalize the well-being of the younger generation. If their interests are not aligned, this can bias the transmission of collective memory towards too little suppression of bad signals (when investments give rise to a negative inter-generational externality) or too much suppression of bad signals (when investments give rise to a positive inter-generational externality). This is consistent with the observation that collective memory is strongly biased towards the suppression of bad signals and rehearsal of good signals in totalitarian regimes (dictators directly value investments which stabilize their regime).

These results apply to the benchmark case of culturally homogeneous societies. The second part of the paper investigates whether and how the insights of the benchmark case need to be modified when we allow for cultural heterogeneity. I modify the benchmark model to study a society with two distinct communities ("cultural groups"), each endowed with its own culture. In this case, each individual has to make two investment decisions: the first concerns "owncultural" investment (learning about and participating in the culture of his own cultural group); the second concerns "cross-cultural" investment (learning about and participating in the culture of the other group). These investments generate two types of externality: the first is the externality exerted by each individual on other members of his own cultural group, and the second is the externality exerted 
by the individual on members of the other cultural group. In order to focus on the implications of cultural heterogeneity, I consider two symmetric communities that differ only in terms of their cultural identity, and study, as before, the transmission of memory by a principal seeking to maximize the welfare of the younger generation, giving equal weight to each community.

The key new issue that arises in this setting concerns the transmission of "mixed news" signals; that is, signals that are "bad news" about one culture and at the same time "good news" about the other. For example, evidence of "bad" behavior by one group can highlight, by contrast, the "good" behavior, or at any rate the absence of bad behavior, of another. Similarly, evidence of the achievements and successes of one group can draw attention to the failures, or simply the lack of achievements, of another. Should this kind of memory be suppressed? I find that the costs of suppression outweigh the benefits: with two symmetric communities, it is always optimal to communicate truthfully "mixed news" signals. Thus the presence of cultural heterogeneity reduces (although it does not eliminate) the scope for welfare-enhancing manipulation of the information transmitted to the young. In particular, the only potentially beneficial departure from truthful communication is associated with the possibility of suppressing memories that are "bad news" for both cultures, or equivalently emphasizing memories that are "good news" for both.

In the presence of cultural heterogeneity, how will collective memory transmission differ from the social optimum in practice? To address this question, I relax the assumption of a social welfare-maximizing principal and assume that the older generation of each community seeks to maximize the welfare of its own community. This is an extreme but convenient simplifying assumption intended to shed light on the possible biases arising when individuals give more weight to their own community in their preferences. I find that as long as each community is able to communicate publicly its information, it will be possible to implement the optimal transmission of "mixed news" signals. This case essentially corresponds to that of a multi-cultural democracy in which each group provides "checks and balances" that limit the scope for other groups to transmit their preferred version of the past. When the underlying signal is "bad news" for both communities, on the other hand, I find that communication will be biased towards too little suppression, unless the communities can implement the social optimum through cooperation to internalize cross-cultural externalities.

The paper proceeds as follows. The remainder of this section discusses the relationship to the existing literature in economics. Section 2 discusses the evidence 
on collective memory and relates it to the model. Section 3 presents the basic version of the model, which is analyzed in section 4 . Section 5 extends the model to study the implications of cultural heterogeneity. Section 6 concludes.

\subsection{Relationship to the literature}

This paper is clearly related to two important literatures within economics. First, the literature on cultural transmission and identity. The closest work to mine is probably Bisin and Verdier (2000, 2001). They study the transmission of cultural traits which results from the interaction of two key influences on the preferences of the young: first, the direct socialization effort of their parents, who wish to transmit their own cultural traits; second, the effect of the broader social and cultural environment, including friends, peers, teachers and others who may act as role models. These influences are not modeled explicitly, however: parents can, at a cost, affect the probability that their children will inherit their cultural traits; if this parental socialization effort fails, children acquire the cultural traits of some role model chosen randomly from the population at large. I view my work as essentially complementary to that of Bisin and Verdier, since it explores one important way in which parents and society at large influence the acquisition of cultural traits by the young. Specifically, I analyze the transmission and manipulation of memory (information about the past). This in turn influences the cultural investment decisions made by the young: it is through this channel that it will ultimately affect their preferences, including their acquisition of cultural traits. While complementary to that of Bisin and Verdier, this approach allows me to identify a different set of important externalities, with quite different welfare implications.

Collective memory is an important determinant of identity: in this sense, the present paper is also related to Akerlof and Kranton (2000), and Bénabou and Tirole (2005). In common with these papers, I view identity as (partly) endogenous. My focus is different from theirs, however, as I investigate how the transmission of collective memory affects cultural investment decisions; these decisions will then shape identity ${ }^{10}$.

The second related literature is the large literature on strategic communication, built on the seminal work of Crawford and Sobel (1982) for the case of soft information, and Grossman (1981), Grossman and Hart (1980) and Milgrom

\footnotetext{
${ }^{10}$ For another related paper which explores the choice between bi-culturalism and monoculturalism, see Lazear (1999).
} 
(1981) for the case of hard information. My paper is more closely related to the second case (the main models I study in sections 4 and 5 are models with hard information $)^{11}$, although there is also a link with the first (I consider a variant with soft information at the end of section 4).

In common with Caillaud and Tirole (2006) and Farrell and Gibbons (1989), I study communication between a sender and multiple receivers. Caillaud and Tirole (2006) focus, as I do, on the transmission of hard information ${ }^{12}$, but their main interest lies in private communication, and especially in persuasion strategies that transmit information to a selectively chosen subset among several receivers. My main interest lies instead in public communication, where the (same) message reaches all receivers. In this respect, my paper is closer to Farrell and Gibbons (1989), who find that, with public communication, the presence of one audience may discipline the sender's communication with the other, leading to full disclosure. Theirs is a model of cheap talk. A similar discipline effect leading to full disclosure of "mixed news" is at work in section 5 of the present paper, but in a very different model with hard information.

Section 5 also considers a version of the model with multiple senders, each sender's preferences being partially congruent with those of one of the receivers. With public communication, this leads again to full disclosure of "mixed news". Full disclosure with multiple senders and a multi-dimensional signal is also obtained in Battaglini (2002); his model is very different though (cheap talk and one receiver), as is the intuition (in his model, it is the multidimensionality of the signal that drives the result; here it is the combination of hard information with the pairwise partial alignment of preferences between senders and receivers).

\footnotetext{
${ }^{11}$ However, the well-known 'unraveling result' of Grossman (1981), Grossman and Hart (1980) and Milgrom (1981), leading to full disclosure of information, does not apply here. This is because, as in Jung and Kwon (1988) for example, agents cannot know whether a "no news" signal corresponds to genuine "no news" or to suppression of "bad news".

${ }^{12}$ More precisely, they focus on the transmission of "semi-hard" information, in the sense that the information is hard (verifiable), but the receiver has to incur a private cost in order to assimilate it.
} 


\section{Collective memory: how societies remember, and forget}

There is a large and growing literature on collective memory ${ }^{13}$, based on research by historians, sociologists and psychologists, that studies the ways in which societies remember, represent and interpret the past. This literature documents the role of school textbooks, newspaper articles, television and radio programs, film, monuments, museums, commemorative rituals, plays, novels, and so on: In other words, the sources that are most likely to impact on the beliefs of the young. Of particular interest from the perspective of the present paper is the wealth of detailed case studies documenting the relationship between collective memory and national identity, for many different countries. These studies provide plenty of evidence that collective memory tends to suppress bad signals. As noted earlier, this occurs in several ways: first, "negative" information is sometimes neglected, ignored and forgotten. Second, it is sometimes presented and interpreted in such a way as to effectively suppress the bad signal. Third, attention is distracted from bad signals by emphasis and elaboration of "positive" information. Given space constraints, it would be impossible for me to summarize all the relevant evidence and examples. I shall therefore discuss a few examples to illustrate the main mechanisms at work, and make it clear that the evidence is not limited to particular time periods, countries, or events; abundant additional evidence can be found in the cited sources.

\subsection{Suppressing bad signals: national origins, values and identity}

Nations often construct and transmit to the young representations of the past intended to provide an attractive account of the nation's cultural values and identity. These are sometimes referred to as "myths", although not in the sense of being either entirely fictional or irrational. "Myth" in this context should be understood as "a set of beliefs, usually put forth as a narrative, held by a community about itself" (Schöpflin (1997), p.19). Notice that this definition does not carry

\footnotetext{
${ }^{13}$ See Olick and Robbins (1998) for a survey, as well as McBride (2001) and Zerubavel (1995) for excellent discussions. Some authors prefer to use the term "social memory" (e.g. Fentress and Wickham (1992)) or "cultural memory" (e.g. Sturken (1997)). The notion that collective memory is to a large extent shaped by, and suited to, current needs and interests, can be traced back to the sociologist Maurice Halbwachs (1925). See also, among others, Buruma (1994); Darian-Smith and Hamilton (1994); Edwards and Middleton (1990); Fabre and O'Meally (1994); Hobsbawm and Ranger (1983); Kammen (1978); Maier (1988); Müller (2002); Olick and Levy (1997); Schwartz (1998); Thelen (1989) and Trouillot (1995), as well as the works cited in the remainder of this section.
} 
any implication of falsehood or invention, and is consistent with the formalization in terms of signal transmission used in this paper. In practice, these "myths", while generally based on historical truth, typically neglect negative information and emphasize positive information. American "myths" of origins, for example, were built around the arrival of the Pilgrim Fathers, the opening of the West, the American Revolution and the Civil War ${ }^{14}$. In each case, the narrative emphasized those aspects that enhanced the appeal of the cultural values that were intended to be transmitted to the young: religious freedom, freedom of expression, progress, self-reliant individualism, democratic equality ${ }^{15}$. Aspects that were less in tune with these values were largely neglected and suppressed ${ }^{16}$.

National "myths", in the sense defined above, have played a very important role in cultural transmission in many countries ${ }^{17}$. In France, for example, the French Revolution provided fertile ground for myth-building ${ }^{18}$, intended to confirm and reinforce the country's "republican values", thereby inducing the young to invest in them. The importance of memory for investments was well recognized by Ernest Renan in 1882, when he argued that "the essence of a nation is that all individuals have many things in common, and also that they have forgotten many things". Chief among the things to be forgotten, in his view, were bad signals about the likely returns to investment in French national identity and norms of cooperation, tolerance and unity: "Every French citizen has to have forgotten the massacre of Saint Bartholomew, or the massacres that took place in the Midi in the thirteenth century" 19 .

\footnotetext{
${ }^{14}$ For suggestive evidence of the impact of such "myths" on the younger generation (in particular, a sample of over 1000 college students), see Frisch (1989).

${ }^{15}$ See, among others, Grant (1997), Hellmann (1986), Kammen (1991), Schwartz (1991).

${ }^{16}$ Thus the myth of the Fathers emphasized the role of English Pilgrims fleeing persecution while ignoring the many who arrived in America for quite different reasons, and downplaying the violence of American colonial society (Grant (1997)). The myth of the West was intimately bound up with the development of the American understanding of democracy (see Schwartz (1991)) and required significant amnesia over aspects of the relationship with Native Americans (see Boime (1998) and Kammen (1991)) and with Mexico (Boime (1998); Kammen (1991)). The myth of the American Revolution (as with many other countries' wars of independence) overstated the degree of consensus prevailing at the time, and largely forgot the fate and number of loyalists (Grant (1997); Kammen (1991)). The suppression of bad signals involved in the construction of the Civil War myth is discussed in detail in Grant (1997).

${ }^{17}$ For a wealth of other examples of national myths, see Eley and Suny (1996), Hosking and Schöpflin (1997) and Samuel (1998).

${ }^{18}$ See Nora ((1992) [1996]) for this and other examples of French myths.

${ }^{19}$ Renan ((1996) [1882], p.45).
} 


\subsection{Suppressing bad signals: collective memories of the Second World War}

Is the tendency for collective memory to suppress bad signals limited to myths of national origins and distant historical events? To answer this question, it is interesting to consider the evidence on a major, relatively recent event that had a profound impact on very many countries: World War II. We find, first, considerable variation among countries. Second, we find nevertheless a clear tendency for collective memory to neglect negative information about the value of investing in national identity and norms of cooperation, fairness, tolerance and unity (e.g. acts of cowardice and betrayal, collaboration with the enemy, bad treatment of prisoners and civilians, massacres, extermination), and emphasize positive information (e.g. acts of courage, resistance against the enemy - and of course negative information about other countries, when this provides a favorable comparison $)^{20}$. This evidence is again consistent with the analysis developed in the present paper.

Moreover, a number of countries experienced a change of political regime as a result of the war, often associated with a radical change in cultural values and norms. Affirmation of these new values required rejection of the old values, and disclosure of "bad signals" about the culture of the old, rejected regime. By highlighting the contrast with the new values, such disclosure effectively sheds a favorable light on the new political culture. In terms of the analysis developed in section 4, this represents another way of fostering optimism about the current (new) culture and encouraging the young to invest in it. The evolution of collective memory in, for example, the former West Germany and the former East Germany is broadly consistent with this analysis ${ }^{21}$. The reality of such a process is of course far more complex than can be captured by any stylized model; in particular, people cannot simply switch their values following a change of political regime. The process of change can be started under the leadership of that part of the older generation that had opposed the values of the old regime, but it will often require the collaboration of many who did not. Thus full disclosure of "bad signals" about the old regime can take some time ${ }^{22}$.

\footnotetext{
${ }^{20} \mathrm{~A}$ wealth of examples can be found, for a large number of countries: see, among others, Boime (1998); Conrad (2003); Hilton and Liu (2005); Judt (1992, 2005); Rousso (1991); Shimazu (2003); von Borries (2003).

${ }^{21}$ See von Borries (2003).

${ }^{22}$ This was the case not only in Germany (see von Borries (2003)), but also in France over Vichy (Rousso (1991)). The French pattern was one of trauma and purges in the immediate aftermath of Vichy, followed by memory suppression and emphasis on the Resistance, followed
} 


\subsection{The evolution of collective memory: George Washington}

The example of countries that experience a radical change of political regime and associated values can be thought of as an extreme case of the more general tendency for collective memory to evolve in line with cultural values, uncovering memories that no longer represent a bad signal, and suppressing memories that now do represent a bad signal, as would be predicted by the analysis in this paper. Again, there is abundant evidence to support this implication. A good example is provided by the evolution of American collective memory of George Washington before and after the Civil War, analyzed in detail by Schwartz (1991). This study shows how collective memory of Washington in the period between his death and the Civil War emphasized his refinement and gentility, his self-restraint and asceticism, his dignity and remoteness - in short, "an aristocratic ideal - an ideal more suited to the gentleman than to the common man, to an eighteenth-century republic than a modern democracy". In the aftermath of the Civil War, Washington's popularity declined and more critical accounts emerged; his aristocratic background became an embarrassment for his democratic supporters. This phase was followed by the construction of a new, more democratic memory of Washington, involving the rediscovery of many of his forgotten traits - his "adeptness at frontier living, his experience at common labor and attachment to the common people, his kindness to children, his strong romantic inclinations" and other "traits that Americans had always associated with democracy". Moreover, the new memory of Washington emphasized that he would have liked to abolish slavery, and was always kind to his slaves. The new memory complemented and democratized the old, making "Washington all the more appealing". This 'democratization' of Washington made it possible to continue to use him as a symbol of American values and a role model for young Americans.

\subsection{The evolution of collective memory: building a new nation}

My last example is the work of Zerubavel (1995) on Israeli collective memory, whose roots are traced back to the Hebrew culture of Palestinian Jews prior to the foundation of the State of Israel in 1948. Zerubavel shows how at this time "the Zionist commemorative narrative accentuated the perception of a 'great divide' between Antiquity and Exile"; specifically, Antiquity was portrayed as a positive period, associated with heroic struggles, and "contrasted with a highly negative

by full disclosure of the "bad signals" associated with the Vichy regime. 
image of Exile", seen as excessively passive in the face of persecution. Jewish memory in Exile was blamed for a "deliberate suppression of the national memory of the ancient struggles for liberation". This made it possible to actively search for a more positive memory of national identity: "The belief in Jewish collective amnesia as far as the national heroic aspects of the past were concerned led to a deliberate Zionist search for suppressed symbols of ancient heroism". Hence, for example, the "rediscovery of Masada" ${ }^{23 "}$ : Zerubavel traces "Masada's odyssey from the periphery of historical chronicles to the center of modern Jewish historical consciousness".

This example further illustrates the tendency for collective memory to suppress bad signals and emphasize good signals. From a Zionist perspective, Jewish memory in Exile represented to some extent a "bad signal", which could be suppressed by questioning its reliability (in part). This opened the way for the reconstruction of a memory which emphasized "good signals": ancient struggles for liberation, as evoked by the memory of Masada - a positive signal for investment in national identity $^{24},{ }^{25}$.

\footnotetext{
${ }^{23}$ This refers to the siege of Jewish rebels at Masada by the Roman army (A.D. 73), culminating with the Jews' collective suicide before the Romans could enter their fortress.

${ }^{24}$ Notice that "collective amnesia" about Masada during Exile is also consistent with the analysis of this paper: Jews in Exile typically had to invest in adopting the national identity of their host countries (while retaining their religious identity). The memory of struggles for independence evoked by Masada would have represented a bad signal in this context, and was largely forgotten. Later on, with the foundation of the State of Israel, the opportunity cost of investing in other national identities increased (an increase in $c$, in terms of the model of section 3 below), leading to a rejection of the previous collective amnesia over Masada among those Jews who chose to invest in a new, Israeli national identity.

${ }^{25}$ The subsequent evolution of collective memory from the 1960s onwards is also interesting. In Zionist commemorative narrative, Masada was remembered as a symbol of active resistance, in contrast with the Jewish experience of Exile. But since then it has also become a symbol of the "great threat to Jewish survival throughout Jewish history, which applies also to the State of Israel". In a nutshell, subsequent Israeli experience changed perceptions, and what had been seen as a "bad signal" in the earlier phase no longer represented a bad signal later on. Consistent with the analysis of this paper, collective memory evolved: Zerubavel found "a greater readiness on the part of Israelis today to accept as their own the Jewish past - not only Antiquity but also the two thousand years of Exile".
} 


\section{The model}

This section introduces the simplest version of the model, which will be used to study the benchmark case in section 4 . This basic version of the model will then be extended to allow for cultural heterogeneity in section 5 .

The model has three dates, $t=0,1,2$, and three players, a "principal" $P$ and two identical "agents", $A_{i}$ and $A_{j}$. The principal represents the first generation ("old"), while the agents represent the second generation ("young"): for simplicity, all players are assumed to be risk-neutral. Information (memory) is transmitted by the principal to the agents at date 0 ; given this information, the agents at date 1 have to make their cultural investment decisions, which may be thought of as investments in learning and internalizing cultural norms and values, as discussed in the Introduction. In what follows, I shall refer to these equivalently as decisions to "invest in the cooperative project", or simply as "effort" decisions. The returns from these investments are realized at date 2 .

The agents' preferences are described by the following utility function:

$$
U_{i}=m x_{i}+g m x_{j}-c x_{i}
$$

where $x_{i} \in\{0,1\}$ denotes agent $A_{i}$ 's investment (effort) decision: if the agent invests in the cooperative project (exerts effort), $x_{i}=1$; if the agent does not invest (exerts no effort), $x_{i}=0$. The variable $m$ represents the value of the culture passed on from the "old" to the "young" generation, which in my definition includes institutions, values, norms, and other such cultural assets (e.g. language) which affect the young generation's ability to succeed in the cooperative project. A higher value of $m$ increases the returns from both agents' investments. The constant $g$ may be positive or negative, depending on whether the society's cultural values and norms tend to generate positive or negative externalities. In much of the analysis that follows, I will focus on the more interesting case where $g$ is strictly positive, so that each agent benefits to some degree from the cooperative investment (effort) of the other agent. It is this positive externality which will create the potential for some welfare-enhancing manipulation of information transmission, as will become clear below. Each agent incurs an effort cost $c$ if he invests in the cooperative project. This may be interpreted literally as an effort cost (e.g. the effort of learning and internalizing cultural norms), but also as an opportunity cost (reflecting the attractiveness of other options, e.g. leisure, crime, learning and adopting different cultural values).

To study the socially optimal transmission of memory, I assume that the prin- 
cipal fully internalizes the agents' welfare. Thus his utility is simply equal to the sum of the agents' individual utilities:

$$
U_{p}=U_{i}+U_{j}=(m+g m-c)\left(x_{i}+x_{j}\right)
$$

The model's information structure is as follows. At date 0 , the principal receives a signal $s$ which is informative about $m$ (e.g. historical evidence informative about how good those institutions and norms really are). I will assume that the principal cannot simply manufacture a good signal (e.g. invent history), but he can suppress a bad signal, as discussed earlier. The assumption that the principal can suppress a bad signal but cannot invent a good signal is motivated by the literature on collective memory, reviewed in section 2. The examples discussed there (e.g. the evolution of American collective memory of George Washington, or the American Revolution and the Civil War; the evolution of Israeli collective memory about Masada) all illustrate the tendency for collective memory to suppress bad signals and emphasize and elaborate good signals, within the constraints imposed by historical records: there is no outright fabrication of good signals.

This is not to say that there is never any fabrication of good signals. Indeed, some historians have emphasized the role of "invented" traditions ${ }^{26}$. Others have argued that in fact collective memory remains rooted in genuine memories much more than would be consistent with the ability to simply "manufacture" remembrance $^{27}$, and that, although outright fabrication does occur sometimes, what occurs more often is "the imaginative reworking of pre-existing materials" 28 . The model developed in this section is intended to capture the latter view, while section 4.2.4 will consider the implications of the former.

For simplicity, I shall focus on the case where $s$ can take just two values: $s=B$ ("bad" signal) and $s=\emptyset$ (no signal). The expected value of $m$ conditional on each possible realization of the true signal $s$ is given by:

\footnotetext{
${ }^{26}$ Hobsbawm and Ranger (1983), for example. Their work focuses particularly on the emergence of the western nation-states in the period 1870-1914, during which a variety of "traditions" (national festivals, symbols and rituals) were established, they argue, to provide a sense of continuity with the past and hence legitimacy for the ruling élites. For example, the institution of Bastille Day dates from this period (1880).

${ }^{27}$ See, among others, Hutchinson (1987), McBride (2001) and Smith (1999). Looking at the evidence from Irish history, McBride argues that the notion of "manufactured" remembrance is difficult to sustain, and finds considerable continuity between genuine, older, informal traditions, and the supposedly "invented" ones that followed them.

${ }^{28}$ McBride (2001).
} 


$$
m_{L}=E[m \mid s=B]<m_{H}=E[m \mid s=\emptyset]
$$

The problem for the young is that they do not observe the signal $s$ before they have to make their effort (investment) decisions: they therefore rely on the information transmitted by the older generation. At the same time, I will assume that they do not rely on such information naively, and they are aware of the possibility that the older generation may manipulate the information it transmits to them in order to manipulate their beliefs and thereby affect their investment decisions. At the end of section 4 I will also consider how the results would be affected if we assumed that the young have naive beliefs.

Let $\hat{s}$ be the signal transmitted by the principal to the agents. Given our assumptions, if the true signal is $s=\emptyset$, there is no opportunity for signal manipulation; thus $\hat{s}=\emptyset$. On the other hand, if the true signal is $s=B$, the principal may either communicate the signal truthfully to the agents $(\hat{s}=B)$, or he may decide to suppress the bad signal $(\hat{s}=\emptyset)$. Given the information transmitted by the principal at date 0 , the agents at date 1 have to make their respective investment decisions. At this date, and before choosing his effort, each agent learns the cost of effort, $c$. I assume that this is not known at date 0 , to allow for possible technology, political and institutional shocks and their impact on the effort/opportunity cost of investing in learning and adopting the society's cultural norms ${ }^{29}$. At date 0 , the effort cost $c$ is known to have a continuous distribution $F(c)$ over the interval $\left[c_{L}, c_{H}\right]$, with density $f(c)>0$. To make the analysis interesting, I will make the following assumption:

$$
c_{L}<m_{L}<m_{H}<c_{H}
$$

implying that, regardless of the information transmitted by the principal, there is a strictly positive ex-ante probability that the agents will exert effort ex post,

\footnotetext{
${ }^{29} \mathrm{An}$ example of a major political shock was the collapse of the Soviet Union. In Russia, this entailed a substantial rise in $c$ during the transition period that followed: poor law enforcement, higher unemployment, frequent wage arrears, rising crime and corruption, all combined to increase the effort/opportunity cost of investment in the existing cultural norms. This was associated with a dramatic increase in alcohol consumption, largely responsible for the sharp decline in life expectancy in 1992-1994 (through alcohol poisoning, heart attacks and strokes, as well as a surge in alcohol-related accidents, violent deaths and suicides. There were an estimated 2.1 million excess deaths between 1992 and 1995; see Rocco (2005) for a detailed case study). Thus for a significant number of people, non-investment or disinvestment took an extreme form, consistent with the sharp rise in $c$.
} 
and a strictly positive ex-ante probability that they will not. ${ }^{30}$ Finally, I assume, for simplicity, that the value of $g$ is public information.

\section{Memory transmission in a culturally homogeneous soci- ety}

This section examines the implications of the benchmark case outlined and motivated in section 3, with a single principal representing the older generation and two identical agents representing the younger generation. Consider to begin with the agents' decisions at date 1 , in the light of the information then available to them. Each agent has to form expectations over the returns from investment, hence over $m$. In doing so, the agents will take into account the possibility that the signal transmitted by the principal may be manipulated, relative to the true signal $s$. Let the agents' prior beliefs concerning the true signal be described by the probability $q$; that is, the agents believe that $s=\emptyset$ with probability $q$ and $s=B$ with probability $1-q$ (these "uninformed" beliefs will be based on whatever information is readily apparent to everyone, including the young). When they receive the principal's signal, $\hat{s}$, the agents have to assess its reliability, based on their beliefs concerning the true signal and their beliefs concerning the communication strategy (truthful or otherwise) used by the principal. Given our assumptions, the principal's communication strategy can be described by the probability $h$ that he will truthfully communicate the bad signal:

$$
h=\operatorname{Pr}[\hat{s}=B \mid s=B]
$$

The agents' beliefs concerning the principal's communication strategy will be denoted by $h^{*}$ : thus a high value of $h^{*}$ corresponds to a high level of "trust" between the older and the younger generation, and conversely a low value of $h^{*}$ implies that the young give a relatively low weight to the information transmitted by the older generation in forming their beliefs. In the limit, when $h^{*}=0$, the young regard the signal transmitted by the old as completely uninformative.

I will assume that the agents update their beliefs according to Bayes' rule, which captures the idea that the young cannot simply be fooled into believing

\footnotetext{
${ }^{30} \mathrm{An}$ interesting alternative would be to consider a version of the model where the younger generation is represented by a continuum of agents with heterogeneous costs, distributed according to $F(c)$. In this case, a fraction of the population would always invest; another fraction would never invest; and finally, a fraction of the population with intermediate costs would invest if, and only if, it held sufficiently optimistic beliefs about the value of $m$.
} 
anything the older generation wishes them to believe (the implications of naive beliefs are considered at the end of the section). Thus if the principal transmits the signal $\hat{s}=\emptyset$, the agents estimate the following probability that the signal is accurate (the signal's "reliability"):

$$
r^{*}=\operatorname{Pr}\left[s=\emptyset \mid \hat{s}=\emptyset ; h^{*}\right]=\frac{q}{q+(1-q)\left(1-h^{*}\right)}
$$

implying that their expected value of $m$ is given by:

$$
m\left(r^{*}\right)=r^{*} m_{H}+\left(1-r^{*}\right) m_{L}
$$

When will the agents provide effort? Given the principal's signal $\hat{s}$, each agent will provide effort (invest in the cooperative project) if, and only if:

$$
E[m \mid \hat{s}] \geqslant c
$$

We can immediately see that the principal may have an incentive to manipulate the information he communicates to the agents by considering his expected utility when the true signal is $s$ and he transmits the signal $\hat{s}$ :

$$
E\left(U_{p} \mid s, \hat{s}\right)=\int_{c_{L}}^{E[m \mid \hat{s}]} 2\{(1+g) E[m \mid s]-c\} d F(c)
$$

If the principal could simply choose the agents' beliefs, he would clearly set them equal to:

$$
E[m \mid \hat{s}]=(1+g) E[m \mid s]
$$

Thus as long as $g>0$, the principal would like the agents to be optimistic about the value of the existing norms and institutions; that is, to form higher expectations than they would if they could observe the true signal $s$. The reason is of course that when $g>0$, each agent's decision to invest in the cooperative project exerts a positive externality on the other agent, but neither agent takes this into account when choosing his effort. If the agents are accurately informed about the true signal, the result is an under-provision of effort relative to the social optimum. By manipulating the agents' beliefs and increasing their confidence in the value of their culture, the principal could correct this under-investment.

Obviously the principal cannot simply choose the agents' beliefs. We must therefore examine the relationship between the principal's communication strategy, $h$, and the agents' beliefs and investment decisions. 


\subsection{What to do with bad signals: disclose or suppress?}

Suppose the principal observes the "bad" signal $(s=B)$ at date 0 . If he transmits the signal accurately to the agents $(\hat{s}=B)$, his expected utility is given by:

$$
S W_{T}\left(m_{L}\right)=\int_{c_{L}}^{m_{L}} 2\left\{(1+g) m_{L}-c\right\} d F(c)
$$

where the subscript $T$ stands for "telling the truth". If on the other hand the principal suppresses the bad signal $(\hat{s}=\emptyset)$, his expected utility depends on the agents' beliefs about the reliability of the principal's signal, $r^{*}$, and is given by:

$$
S W_{S}\left(m_{L}, r^{*}\right)=\int_{c_{L}}^{m\left(r^{*}\right)} 2\left\{(1+g) m_{L}-c\right\} d F(c)
$$

where the subscript $S$ stands for "suppression". The net gain from suppressing the bad signal is therefore equal to:

$$
S W_{S}\left(m_{L}, r^{*}\right)-S W_{T}\left(m_{L}\right)=\int_{m_{L}}^{m\left(r^{*}\right)} 2\left\{(1+g) m_{L}-c\right\} d F(c)
$$

If $m\left(r^{*}\right)>(1+g) m_{L}>m_{L}$, the net gain can be written as follows:

$$
\int_{m_{L}}^{(1+g) m_{L}} 2\left\{(1+g) m_{L}-c\right\} d F(c)-\int_{(1+g) m_{L}}^{m\left(r^{*}\right)} 2\left\{c-(1+g) m_{L}\right\} d F(c)
$$

The first integral represents the gains from suppressing the bad signal: by inducing greater optimism about the value of the existing norms and institutions, and hence about the returns from investment, suppressing the bad signal elicits more effort and thereby corrects the under-provision of effort due to the presence of externalities between the agents. However, optimism can go too far: the second integral represents the loss from excessive optimism, which leads agents to invest too much.

The net gain from suppressing the bad signal is clearly increasing in $g$, the magnitude of the externalities between the two agents, and decreasing in $r^{*}$, the agents' beliefs about the reliability of the principal's signal. Thus when the agents' "trust" is high (high value of $r^{*}$ ), the principal's net gain from suppressing the bad signal tends to be lower, because there is a greater danger of excessive optimism: this suggests the possibility of multiple equilibria with different degrees of trust. On the other hand, for sufficiently high values of $g$ (sufficiently important 
externalities), the net gain from manipulating information will always be strictly positive, irrespective of the agents' beliefs: in this case "high trust" equilibria cannot be sustained. The intuition just outlined is confirmed by Proposition 1 below, which characterizes the set of Perfect Bayesian equilibria (PBEs) ${ }^{31}$.

Proposition 1 There exist $g_{H}$ and $g_{L}$, with $g_{H}>g_{L}>0$, such that:

(i) For all $g>g_{H}$, there is a unique PBE with $h^{*}=0$;

(ii) For all $g<g_{L}$, there is a unique PBE with $h^{*}=1$;

(iii) For all $g \in\left[g_{L}, g_{H}\right]$, there are three PBEs: (a) $h^{*}=0$, (b) $h^{*}=1$, and (c) $h^{*}=h(g)$, where $h(g)$ increases from 0 to 1 as $g$ increases from $g_{L}$ to $g_{H}$.

Proof: see Appendix 1.

Proposition 1 confirms our intuition concerning the possibility of multiple equilibria: for intermediate values of $g$, both "high-trust" $\left(h^{*}=1\right)$ and "low-trust" $\left(h^{*}=0\right)$ equilibria are feasible, as well as equilibria with an intermediate degree of trust. For high values of $g$, on the other hand, only low-trust equilibria are feasible: when the externalities between the two agents are sufficiently important, the only credible strategy for the principal is to suppress the bad signal. For sufficiently low values of $g$, the opposite is true: the loss from over-investment would exceed any gain from the correction of under-investment; thus the principal's optimal strategy is always to tell the truth, and the only equilibria are high-trust equilibria.

\subsection{Interpretation and extensions}

The results so far have a number of interesting implications for the transmission of collective memory. First, we have shown that if the older generation seeks to maximize the welfare of the younger generation, it will choose to suppress bad signals when the positive externalities generated by individual decisions to invest in the cooperative project are sufficiently important. Thus "telling the whole truth" is not always an optimal strategy: in some cases it is better to manipulate the information transmitted to the young in order to foster optimism about the value of existing cultural norms and institutions and hence about the returns from cultural investment. This leads to a higher provision of effort than would be the case with truthful information transmission, thereby counteracting the tendency

\footnotetext{
${ }^{31}$ Details of the principal's optimization problem, as well as the definition of PBE, are relegated to Appendix 2 for ease of exposition.
} 
for individuals to under-invest because they do not take into account the positive externalities their investment exerts on others.

A number of other implications of the analysis are discussed below together with possible extensions.

\subsubsection{Trust}

Another implication of the results summarized in Proposition 1 is that low-trust equilibria may be better than high-trust equilibria in terms of welfare. For intermediate values of $g$, there exist multiple equilibria, with low-trust equilibria which are strictly better in terms of social welfare than the corresponding hightrust equilibria (i.e. holding the value of $g$ constant) ${ }^{32}$. Thus a high degree of scepticism on the part of the young concerning the reliability of the information transmitted by the older generation need not mean that the society is trapped in a "bad" (low social welfare) equilibrium.

However, this can only be the case if the information available to the older generation represents a "bad" signal $(s=B)$, as assumed in this section so far. The implications of a high or low level of trust are of course very different when the information available to the older generation represents a "good" signal $(s=\emptyset)$. In this case, it is easy to verify that, for any given value of $g \geqslant 0$, social welfare when trust is high $\left(h^{*}=1\right)$ is strictly higher than social welfare when trust is low $\left(h^{*}=0\right)$. Distrust is costly in this case because it means that the young give no weight to the (truthful) good signal, and as a consequence invest too little: I shall refer to this situation as one of "cultural pessimism" 33 .

\footnotetext{
${ }^{32}$ The intuition is that, although there are gains from making the young more optimistic, these gains will be more than offset by the costs of excessive optimism if the young are very trusting: in this case truth-telling is the optimal strategy. If the young are more sceptical, the costs of excessive optimism will be correspondingly lower, and suppressing the bad signal becomes optimal, leading to higher social welfare.

${ }^{33}$ The welfare analysis in this section is conditional on the realization of the signal $s$, which is appropriate as long as each generation has sufficient discretion over its communication strategy with the following generation. This seems a very plausible assumption in many ways: each generation writes its own textbooks, novels and newspaper articles, produces its own films and television programs, and has considerable discretion in ignoring, selectively remembering and interpreting older sources. Indeed, the examples discussed in section 2 are consistent with this assumption.
} 


\subsubsection{Naive beliefs}

So far I have assumed that the younger generation is not naive: it realizes that the older generation may not always tell the truth. This seems a reasonable assumption for adolescents and young adults. On the other hand, it may be argued that the assumption is less compelling for children; moreover, a substantial part of an individual's investment in learning and internalizing cultural values occurs during childhood. It is therefore important to examine how the results obtained above are modified if we assume that the younger generation simply believes the information it receives from the older generation. We have the following result:

Proposition 2 Suppose the agents always believe the principal is telling the truth. Then:

(i) For all $g>g_{H}$, the principal will suppress the bad signal $(h=0)$;

(ii) For all $g \leqslant g_{H}$, the principal will tell the truth $(h=1)$.

Proof: see Appendix 1.

Thus with naive beliefs, the principal will still suppress bad signals when social externalities are sufficiently large. However, the assumption of naive beliefs eliminates the multiplicity of equilibria for intermediate values of $g$ (between $g_{L}$ and $\left.g_{H}\right)$ : in this range, the net gain from suppressing bad signals would be negative because the agents are so trusting that they would become too optimistic and invest too much. Thus the principal's optimal strategy is to tell the truth. Truthful communication is of course the optimal strategy also for lower values of $g$.

Overall, naive beliefs modify our earlier results in two important ways. First, they remove the multiplicity of equilibria for intermediate values of the externality parameter $g$ and ensure that truthful communication of bad signals will occur in this range. Second, they remove the costs of distrust and cultural pessimism when the underlying signal is good.

\subsubsection{Fabrication of good signals}

The model studied so far is intended to capture the view, motivated in section 3 , that collective memory can suppress a bad signal (e.g. through selective remembering) but cannot manufacture a good signal. We can also consider the implications of the view that allows for outright fabrication. The simplest modification of the model that can be used to look at this possibility is a cheap talk version with two possible signals, $s \in\{\emptyset, G\}$, where $\emptyset$ represents "no news" and 
$G$ represents "good news", and $m_{L}=E[m \mid s=\emptyset]<m_{H}=E[m \mid s=G]$. Since the information is soft, the principal can transmit either signal, irrespective of the realization of $s$; in particular, he can "manufacture" a good signal $(s=\emptyset$ and $\hat{s}=G)$. The results are qualitatively similar, in the following sense. For $g \geqslant 0$, a version of Proposition 1 holds, the only difference being that the suppression of bad signals is now replaced by the manufacture of good signals. The intuition is that for $g>0$ the principal will again have an incentive to influence the agents' beliefs so as to make them more optimistic. Thus he will never be interested in transmitting signal $\hat{s}=\emptyset$ when the truth is $s=G$, since this would have the opposite effect: the only manipulation of interest is the transmission of $\hat{s}=G$ when $s=\emptyset$, which will occur in the same circumstances as the suppression of bad signals in the model with hard information ${ }^{34}$. It is therefore possible to accommodate the fabrication of good signals within a simple variant of the basic model, yielding similar insights concerning the transmission of collective memory.

\subsubsection{The role of parents}

When the positive externalities associated with the younger generation's investment decisions are sufficiently important, we have seen that it can be socially optimal to foster optimism among the young. In these circumstances, parents will have a collective interest in suppressing bad signals. Yet their individual interests could differ: in particular, a parent who cares mainly about his/her own children could have an incentive to communicate bad signals to them, privately, while publicly supporting the collective suppression of bad signals. There are certainly cases where parents publicly voice support for, say, cultural norms of honesty, justice, fairness, equal opportunities, openness, and so on, while privately discouraging their own children from taking the risks associated with, for example, fighting corruption, denouncing wrong-doing, or helping victims. However, the extent to which parents individually can undermine the collective suppression of bad signals will be limited by a number of factors, including the following. First, it is difficult to ensure that children will not reveal to anyone what they learn from their parents, and parents may well prefer not to run the risk of being stigmatized (along

\footnotetext{
${ }^{34}$ The two models yield slightly different implications, on the other hand, in the less interesting case where $g<0$. In this case the principal would like the agents to be pessimistic. In the model with hard information this always induces truth-telling, since by assumption the principal cannot manufacture a bad signal. In the model with soft information, the desire to induce pessimism may lead to the suppression of good signals.
} 
with their children). ${ }^{35}$ Second, it can be difficult for individual parents to obtain all the hard information needed to convince their own children, when the social environment (and the parents themselves, in public) are transmitting a different message $^{36}$.

\section{Memory transmission in a multi-cultural society}

The benchmark analysis developed in section 4 assumed a culturally homogeneous society. It is therefore of considerable interest to extend the model and investigate which insights continue to hold and which need to be modified, and how, once we allow for a multi-cultural society.

\subsection{The model: modifications}

To focus on the implications of cultural heterogeneity, I retain the basic structure of the model of section 3 but relax the assumption of homogeneity. The two agents, $A_{i}$ and $A_{j}$, are no longer assumed to be identical: each agent belongs to a different community or "cultural group" (has a different "cultural identity"). The agents' preferences are described by the following utility function:

$$
U_{i}=m_{i} x_{i i}+b m_{j} x_{i j}+g m_{i} x_{j i}-c\left(x_{i i}+x_{i j}\right)
$$

where $m_{i}$ and $m_{j}$ represent the norms, values and institutions of the two cultural groups, respectively. Each agent now has to take two decisions: how much to invest in his own culture (that of his cultural group), and how much to invest in the other culture. For agent $A_{i}$ (agent $\left.A_{j}\right)$, denote by $x_{i i}\left(x_{j j}\right)$ the effort he devotes to learning about and participating in his own culture, and by $x_{i j}\left(x_{j i}\right)$ the effort he devotes to learning about and participating in the other culture. The net benefits from investing in a given culture will depend partly on that culture's norms, values and institutions (as in sections 3 and 4), and partly on the cultural identity of the agent who invests. Thus if agent $A_{i}$ invests in culture $j$, he may

\footnotetext{
${ }^{35}$ The literature on social psychology provides abundant evidence of the importance of social motivation (wanting to establish their social identity and maintain their relations with their peers) for individual behavior. For a review and discussion see Miller and Prentice (1996).

${ }^{36}$ Parents may also prefer not to transmit bad signals to their children for more selfish reasons, as noted in the Introduction (they may obtain psychological as well as practical benefits from their children's investments). Another possibility is that they may wish to help their children overcome a problem of time inconsistency (under-investment due to hyperbolic discounting).
} 
face higher costs and/or reap lower benefits than if agent $A_{j}$ makes an equivalent investment: for example, because agent $A_{j}$ 's parents belong to cultural group $j$, and can more easily communicate knowledge of their own culture to their child (thereby reducing the agent's learning costs), and because agent $A_{j}$ has greater opportunities to interact with members of cultural group $j$ (which reduces his learning costs and increases his benefits from investing in culture $j$ ).

This possible difference is captured by the parameter $b \geqslant 0$ : if $b<1$, there is a comparative disadvantage in investing in a culture other than one's own; the disadvantage disappears for $b=1$. Clearly the value of $b$ can be affected by a variety of policies; in what follows I assume that $b$ is given exogenously, in order to focus on the transmission of memory given $b$. Similarly the effort (opportunity) cost $c$ is assumed to be given exogenously. Moreover, I assume that ex ante the cost is uniformly distributed over the interval $[0,1]$ for both agents (i.e. both cultural groups), in order to have as much symmetry as possible between the two agents (groups). This will provide a clear benchmark analysis, and allow me to focus on the implications of asymmetric signals in an otherwise symmetric setting, as discussed below. Finally, the parameter $g$ now captures the externality exerted by each agent on the other when it invests in the other's culture. To make the analysis interesting, I assume that this externality is positive.

To investigate the welfare-maximizing transmission of memory in the presence of cultural heterogeneity, I shall assume that the principal gives equal weight to each cultural group. His utility will be given by the following expression:

$$
U_{p}=U_{i}+U_{j}+V m_{i} x_{i i}+V m_{j} x_{j j}
$$

The principal therefore maximizes the sum of the two agents' utilities, plus two terms reflecting the fact that each agent's decision to invest in his own culture exerts an externality, represented by $V$, on other members of his cultural group. This is a convenient way of allowing for the intra-group externalities that were studied in section 4 without explicitly modeling the interaction of agents within the same cultural group as well as across cultural groups. I focus on the more interesting case where these externalities are positive.

The information structure of the model is modified as follows. $P$ (the principal) now receives a two-dimensional signal, $s=\left[s_{i}, s_{j}\right]$, where $s_{i}$ is informative about the value of $m_{i}$ and $s_{j}$ is informative about the value of $m_{j}$. I allow for the possibility of "bad news", "no news" and "good news"; thus $s_{k} \in[\emptyset, B, G](k=$ $i, j)$. The expected value of $m_{k}(k=i, j)$, conditional on each possible realization of $s_{k}$, is given by: 


$$
m_{L}=E\left[m_{k} \mid s_{k}=B\right]<m_{M}=E\left[m_{k} \mid s_{k}=\emptyset\right]<m_{H}=E\left[m_{k} \mid s_{k}=G\right]
$$

where $0<m_{L}<m_{H}<1$. I assume that "good news" and "bad news" are symmetric relative to "no news", in the sense that $m_{L}$ and $m_{H}$ are equidistant from $m_{M}$ :

$$
m_{L}+z=m_{M}=m_{H}-z
$$

for some $z>0$.

As noted in the Introduction, the key new issue that arises in this context concerns the principal's communication strategy in the presence of "mixed news": that is, a signal which is good news about one culture but simultaneously bad news about the other. To investigate the issue of mixed news, I shall focus on the case where $s$ can take just four possible values: $s \in\{[\emptyset, \emptyset],[B, B],[B, G],[G, B]\}$. Thus the principal may receive no signal about either $m_{i}$ or $m_{j}$; he may receive a bad signal about both, or he may receive a signal which is bad for one and good for the other. This is the simplest framework I can use to study the issues of interest. I continue to assume that the principal cannot simply manufacture a signal; on the other hand he can suppress a signal. Thus if the true value of $s$ is $[\emptyset, \emptyset]$, the principal has no scope for manipulating the information he transmits to the agents: $\hat{s}=[\emptyset, \emptyset]$. However, if the principal receives a signal $s \in\{[B, B],[B, G],[G, B]\}$, he can either communicate the signal truthfully to the agents, or suppress it (in the latter case, $\hat{s}=[\emptyset, \emptyset])$.

The principal's communication strategy therefore consists of three probabilities, $h_{B B}, h_{G B}$ and $h_{B G}$, defined by:

$$
\begin{aligned}
& h_{B B}=\operatorname{Pr}\{\hat{s}=[B, B] \mid s=[B, B]\} \\
& h_{B G}=\operatorname{Pr}\{\hat{s}=[B, G] \mid s=[B, G]\} \\
& h_{G B}=\operatorname{Pr}\{\hat{s}=[G, B] \mid s=[G, B]\}
\end{aligned}
$$

As for beliefs, I assume full symmetry between the agents ex ante (again, as a benchmark); that is, they share the same "uninformed" beliefs before they receive the principal's signal ${ }^{37}$.

\footnotetext{
${ }^{37}$ Further details about this version of the model can be found in Appendix 2.
} 


\subsection{Multi-cultural societies: mixed-news signals}

Suppose the principal receives a mixed-news signal; for example, $s=[G, B]$. If he communicates the signal truthfully to the agents, his expected utility is equal to:

$$
\begin{gathered}
S W_{T}\left(m_{H}, m_{L}\right)=\int_{0}^{m_{H}}\left[m_{H}(1+V)-c\right] d c+\int_{0}^{m_{L}}\left[m_{L}(1+V)-c\right] d c \\
+\int_{0}^{b m_{L}}\left[(b+g) m_{L}-c\right] d c+\int_{0}^{b m_{H}}\left[(b+g) m_{H}-c\right] d c
\end{gathered}
$$

If the principal suppresses the true signal, his expected utility is given instead by the following expression:

$$
\begin{gathered}
S W_{S}\left(m_{H}, m_{L}\right)=\int_{0}^{m_{i}\left(r^{*}\right)}\left[m_{H}(1+V)-c\right] d c+\int_{0}^{m_{j}\left(r^{*}\right)}\left[m_{L}(1+V)-c\right] d c \\
+\int_{0}^{b m_{j}\left(r^{*}\right)}\left[(b+g) m_{L}-c\right] d c+\int_{0}^{b m_{i}\left(r^{*}\right)}\left[(b+g) m_{H}-c\right] d c
\end{gathered}
$$

Thus the net gain from suppressing the mixed-news signal is equal to:

$$
\begin{gathered}
S W_{S}\left(m_{H}, m_{L}\right)-S W_{T}\left(m_{H}, m_{L}\right) \\
=-\int_{m_{i}\left(r^{*}\right)}^{m_{H}}\left[m_{H}(1+V)-c\right] d c+\int_{m_{L}}^{m_{j}\left(r^{*}\right)}\left[m_{L}(1+V)-c\right] d c \\
+\int_{b m_{L}}^{b m_{j}\left(r^{*}\right)}\left[(b+g) m_{L}-c\right] d c-\int_{b m_{i}\left(r^{*}\right)}^{b m_{H}}\left[(b+g) m_{H}-c\right] d c
\end{gathered}
$$

The first integral in expression (5.10) represents the effect on agent $A_{i}$ 's decision to invest in his own culture: suppressing the true signal, which was "good news" about the value of $m_{i}$, reduces $A_{i}$ 's incentives to invest, leading to an underprovision of effort. This term therefore represents a net loss from the suppression of the true signal. The second integral shows the effect on agent $A_{j}$ 's decision to invest in his own culture: suppressing the true signal, which was "bad news" about $m_{j}$, increases his incentives to invest, which mitigates the under-provision 
of effort due to the presence of positive externalities $(V>0)$ among members of $A_{j}$ 's cultural group. However, if $m_{j}\left(r^{*}\right)>(1+V) m_{L}$, there will be an overprovision of effort. The net gain from this second effect is therefore analogous to the one examined earlier, in section 4 , and is increasing in $V$, the magnitude of the intra-group externalities.

There are two additional "cross-cultural" effects. The last two terms of expression (5.10) show the effect on each agent's decision to invest in the other agent's culture. Suppressing the true signal increases $A_{i}$ 's cross-cultural investment and reduces $A_{j}$ 's cross-cultural investment. If we assume that $b m_{j}\left(r^{*}\right)>(b+g) m_{L}$, we can write these two terms as follows:

$$
\begin{gathered}
\int_{b m_{L}}^{(b+g) m_{L}}\left[(b+g) m_{L}-c\right] d c-\int_{(b+g) m_{L}}^{b m_{j}\left(r^{*}\right)}\left[c-(b+g) m_{L}\right] d c \\
-\int_{b m_{i}\left(r^{*}\right)}^{b m_{H}}\left[(b+g) m_{H}-c\right] d c
\end{gathered}
$$

This makes clear the different effects at work. The first integral represents the gain from agent $A_{i}$ 's greater optimism about the other culture, which corrects the tendency to under-invest due to the presence of positive cross-cultural externalities. The second integral represents the loss from $A_{i}$ 's excessive optimism about the other culture, which leads him to over-invest. Finally, the last integral represents the loss from agent $A_{j}$ 's under-investment in the other culture.

The principal's optimal communication strategy turns out to be very simple:

Proposition 3 Suppose the principal receives the signal $s=[G, B]$. Then he will always communicate the signal truthfully to the agents : $\hat{s}=[G, B]$ (i.e. $\left.h_{G B}=1\right)$.

Proof: see Appendix 1.

Given the symmetry of the problem, the same obviously applies when the principal receives the signal $s=[B, G]$ : in this case, $\hat{s}=[B, G]$. Thus the principal's optimal communication strategy when he receives a "mixed news" signal is to transmit the signal truthfully to the agents. It follows that the only circumstances in which there is a potential for beneficial manipulation of the information transmitted to the agents are those corresponding to receipt of a signal which is "bad news" for both cultures, i.e. $s=[B, B]$. In this case, as in the mono-cultural case analyzed in section 4 , suppression of the true signal can be welfare-enhancing, provided social externalities (within each cultural group and between cultural groups) are sufficiently important. ${ }^{38}$ 


\subsection{Interpretation and extensions}

The intuition for the result summarized in Proposition 3 is that the productivity of investment is higher for the culture with the higher value of $m$; thus underinvestment in that culture is more costly in terms of social welfare than underinvestment in the other culture. Accurate public transmission of the true signal $s$ is therefore needed to provide efficient investment incentives to both agents.

\subsubsection{Multi-cultural versus mono-cultural societies}

An important implication of this result is that cultural heterogeneity reduces the scope for welfare-enhancing manipulation of memory, in the following sense: a bad signal for one community's culture, which might have been worth suppressing from the perspective of that community, is no longer worth suppressing if it also represents a good signal for the other community's culture.

The analysis in this section shows how the insights developed in section 4 need to be modified to take account of the implications of cultural heterogeneity. On the one hand, the insights of section 4 continue to apply, as might be expected, in the presence of signals that are the same for both cultures. On the other hand, there are circumstances ("mixed news") in which it cannot be optimal any longer to manipulate memory.

\subsubsection{Memory transmission in practice}

So far we have focused on the optimal transmission of memory in the presence of cultural heterogeneity. How will collective memory transmission differ from the social optimum in practice? Clearly if the older generation of each community seeks to maximize social welfare, the outcome will be the same as with the welfaremaximizing principal assumed above. Suppose instead that the older generation

\footnotetext{
${ }^{38}$ Collective memory is transmitted to the majority of the young through channels such as school textbooks, popular histories and biographies, television, radio, novels, movies, internet, monuments, museums, public rituals, and so on. In some cases, though, children grow up in highly segregated communities, with very limited access to such channels. These communities may then be able to suppress, for their own children, a "mixed news" signal which is not favorable to their culture. Such a targeted suppression, leading to higher own-cultural and lower crosscultural investment, can be welfare-enhancing when $V$ is sufficiently large and $b$ and $g$ sufficiently small. However, when $V$ is small (low intra-group externalities) and $b$ large (high returns to cross-cultural investments), targeted suppression will be detrimental for the community itself, as well as for society at large.
} 
of each community is only concerned with maximizing the welfare of its own community. This is of course an extreme but convenient simplifying assumption to investigate the biases that are likely to arise when individuals give a higher weight in their preferences to their own community. Consider the case of a multicultural democracy, in which the older generation of each community receives the true signal $s$ and has the opportunity to communicate the signal publicly.

There are two possibilities of interest. First, the true signal is "bad news" for both cultures (i.e. $s=[B, B]$ ). In this case, the outcome of any Nash equilibrium of the communication game will be biased towards too little suppression of the signal, relative to the social optimum. This is because of the presence of positive cross-cultural externalities $(g>0)$. The second possibility of interest is that of "mixed news". In this case, truthful communication is the (unique) equilibrium outcome of the communication game. The reason is that telling the truth is a dominant strategy for the community whose "own" signal is "good news". In this case, therefore, it is possible to implement the social optimum.

\section{Conclusions}

This paper studies the intergenerational transmission of knowledge about the past, focusing on its implications for cultural transmission and cultural investments by the young. It shows that some manipulation of the information transmitted to the young (the suppression of bad signals) may at times be beneficial, by fostering optimism about the value of existing cultural norms and institutions and thereby encouraging investments which generate important social externalities. This can be seen as providing a rationale for certain biases documented by sociologists, psychologists and historians working on collective memory. However, the ability to bias the transmission of collective memory in this way also has important costs: it may generate cultural over-optimism and over-investment in some cases, and the reverse, cultural pessimism and under-investment, in other cases. Moreover, the scope for beneficial manipulation of information by the older generation is significantly reduced, although not eliminated, in multi-cultural societies, where the truthful disclosure of "mixed-news" signals is needed to provide efficient owncultural and cross-cultural investment incentives.

These findings begin to address some of the policy issues raised in the Introduction. For example, how should history be taught in schools? We have seen that in some circumstances there may be a case for the selective emphasis of good signals and suppression of bad signals; on the other hand, with symmetric com- 
munities, the suppression of "mixed news" signals is never optimal. In practice, many of the signals that one group or another might like to suppress can be interpreted as "mixed news" in an increasingly multi-cultural context (as evidenced for example in the recent controversy over Japanese history textbooks). The scope for welfare-enhancing suppression of information is correspondingly limited. This can raise difficult issues of balance, given that some selection is always necessary.

The problem of balance is also very relevant to the debate on public service broadcasting. The analysis in this paper suggests an important role for public service broadcasting in granting opportunities for public communication to different cultural groups. From this perspective, "balance" requirements ${ }^{39}$ may be valuable as a commitment device to avoid the perception (and/or reality) of costly biases. At the same time, their implementation can raise difficulties, as noted above with regard to classroom teaching and textbooks, because of the need for selection.

Much work remains to be done on these and other related issues. It would be interesting to extend the analysis of this paper in a number of ways. From both a theoretical and a policy perspective, it would be useful to consider asymmetric cultural groups. Another important avenue for further research would be the dynamics of memory transmission and cultural investments, and the role of peer effects. Yet another issue that clearly deserves attention is the relationship between memory and conflict.

\section{References}

[1] Akerlof, G.A. and R.E. Kranton (2000), "Economics and identity", Quarterly Journal of Economics, 115(3), 715-53.

[2] Battaglini, M. (2002), "Multiple referrals and multidimensional cheap talk", Econometrica, 70(4), 1379-1401.

[3] Bénabou, R. and J. Tirole (2002) "Self-confidence and personal motivation", Quarterly Journal of Economics, 117(3), 871-915.

[4] Bénabou, R. and J. Tirole (2006) "Belief in a just world and redistributive politics", Quarterly Journal of Economics, 121(2), 699-746.

[5] Bénabou, R. and J. Tirole (2005) "A cognitive theory of identity", paper presented at the Toulouse conference in tribute to Jean-Jacques Laffont.

[6] Bisin, A. and T. Verdier (2000), "Beyond the melting pot": cultural transmission, marriage, and the evolution of ethnic and religious traits", Quarterly

\footnotetext{
${ }^{39} \mathrm{As}$ in the case of the CPB, for example.
} 
Journal of Economics, 115(3), 955-88.

[7] Bisin, A. and T. Verdier (2001), "The economics of cultural transmission and the dynamics of preferences", Journal of Economic Theory, 97(2), 298-319.

[8] Boime, A. (1998), The Unveiling of the National Icons, Cambridge University Press.

[9] Buruma, I. (1994), The Wages of Guilt: Memories of War in Germany and Japan. New York: Farrar, Straus, Giroux.

[10] Caillaud, B. and J. Tirole (2006), "Consensus building: how to persuade a group", mimeo, University of Toulouse.

[11] Compte, O. and A. Postlewaite (2004) "Confidence-enhanced performance", American Economic Review, 94(5), 1536-57.

[12] Conrad, S. (2003), "Entangled memories: versions of the past in Germany and Japan, 1945-2001", Journal of Contemporary History, 38(1), 85-99.

[13] Crawford, V.P. and J. Sobel (1982), "Strategic information transmission", Econometrica, 50(6), 1431-1451.

[14] Dunning, D. (2001) "On the motives underlying social cognition", in A. Tesser and N. Schwarz (eds.) Blackwell Handbook of Social Psychology: Intraindividual Processes.

[15] Darian-Smith, K. and P. Hamilton (eds.) (1994), Memory and History in Twentieth-Century Australia. Melbourne: Oxford University Press.

[16] Edwards, D. and D. Middleton (eds.) (1990), Collective Remembering (London).

[17] Fabre, G. and R. O'Meally (eds.) (1994), History and Memory in AfricanAmerican Culture. New York: Oxford University Press.

[18] Falk, A. and C. Zehnder (2006), "Discrimination and in-group favoritism in a citywide trust experiment", paper presented at the ENABLE conference, Fraueninsel.

[19] Farrell, J. and R. Gibbons (1989), "Cheap talk with two audiences", American Economic Review, 79(5), 1214-1223.

[20] Fentress, J. and C. Wickham (1992), Social Memory (Oxford).

[21] Frisch, M. (1989), "American history and the structures of collective memory: a modest exercise in empirical iconography", Journal of American History, 75(4), 1130-1155.

[22] Grant, S.-M. (1997), "Making history: myth and the construction of American nationhood", in G. Hosking and G. Schöpflin (eds.) Myths and Nationhood, London. 
[23] Grossman, S.J. (1981), "The informational role of warranties and private disclosure about product quality", Journal of Law and Economics, 24(3), 461-483.

[24] Grossman, S.J. and O. Hart (1980), "Disclosure laws and takeover bids", Journal of Finance, 35, 323-334.

[25] Halbwachs, M. (1925), Les Cadres Sociaux de la Mémoire (On Collective Memory, ed. and trans. L.A. Coser (Chicago, IL, 1992)).

[26] Hellmann, J. (1986), American Myth and the Legacy of Vietnam, New York: Columbia University Press.

[27] Hilton, D. J. and J.H. Liu (2005), "How the past weighs on the present: Social representations of history and their role in identity politics", British Journal of Social Psychology, forthcoming.

[28] Hobsbawm, E. and T. Ranger (eds.) (1983), The Invention of Tradition, Cambridge University Press.

[29] Hutchinson, J. (1987), The Dynamics of Cultural Nationalism: The Gaelic Revival and the Creation of the Irish Nation State, London.

[30] Judt, A. (1992), "The past is another country: myth and memory in postwar Europe", Daedalus 121(4), 83-118.

[31] Judt, A. (2005), Postwar: A History of Europe Since 1945, Penguin.

[32] Jung, W. and Y.K. Kwon (1988), "Disclosure when the market is unsure of information endowment of managers", Journal of Accounting Research, 26(1), 146-153.

[33] Kammen, M. (1978), A Season of Youth: The American Revolution and the Historical Imagination. New York: Knopf.

[34] Kammen, M. (1991), Mystic Chords of Memory: The Transformations of Tradition in American Culture. New York: Knopf.

[35] Lazear, E.P. (1999), "Culture and language", Journal of Political Economy, 107(6), S95-126.

[36] Maier, C.S. (1988), The Unmasterable Past: History, Holocaust, and German National Identity. Cambridge, MA: Harvard University Press.

[37] McBride, I. (2001), "Memory and national identity in modern Ireland", in I. McBride (ed.) History and Memory in Modern Ireland, Cambridge University Press.

[38] Milgrom, P.R. (1981), "Good news and bad news: representation theorems and applications", Bell Journal of Economics, 12(2), 380-391.

[39] Miller, D.T. and D.A. Prentice (1996), "The construction of social norms and standards", in E.T. Higgins and A.W. Kruglanski (eds.) Social psychology: handbook of basic principles, The Guilford Press, New York. 
[40] Müller, J.-W. (ed.) (2002), Memory and Power in Post-War Europe, Cambridge University Press.

[41] Nora, P. (1992), Les Lieux de Mémoire, Paris (Realms of Memory, English language edn (New York, 1996)).

[42] Olick, J. K. and D. Levy (1997), "Collective memory and cultural constraint: Holocaust myth and nationality in German politics", American Sociological Review, 62, 921-936.

[43] Olick, J. K. and J. Robbins (1998), "Social memory studies: from "collective memory" to the historical sociology of mnemonic practices", Annual Review of Sociology, 24, 105-40.

[44] Renan, E. (1882), Qu'est-ce qu'une nation?, Paris (What Is a Nation?, English language version reprinted in G. Eley and R.G. Suny (eds.), Becoming National, Oxford University Press: 1996)

[45] Rocco, L. (2005), Essays on the Political Economy of Mass Mortality, doctoral thesis, University of Toulouse.

[46] Rousso, H. (1991), The Vichy Syndrome: History and Memory in France Since 1944. Transl. A Goldhammer. Cambridge, MA: Harvard University Press.

[47] Samuel, R. (1998), Island Stories: Unravelling Britain, Theatres of Memory, Volume II (London).

[48] Schöpflin, G. (1997), "The functions of myth and a taxonomy of myth", in G. Hosking and G. Schöpflin (eds.) Myths and Nationhood, London.

[49] Schwartz, B. (1991), "Social change and collective memory: the democratization of George Washington", American Sociological Review, 56, 221-236.

[50] Schwartz, B. (1998), "Postmodernity and historical reputation: Abraham Lincoln in late twentieth-century American memory", Social Forces, 77, 63-103.

[51] Shimazu, N. (2003), "Popular representations of the past: the case of postwar Japan", Journal of Contemporary History, 38(1), 101-116.

[52] Smith, A.D. (1999), Myths and Memories of the Nation, Oxford.

[53] Sturken, M. (1997), Tangled Memories: The Vietnam War, The Aids Epidemic, and the Politics of Remembering (Berkeley).

[54] Thelen, D. (ed.) (1989), Memory and American History. Bloomington: Indiana University Press.

[55] Trouillot, M.R. (1995), Silencing the Past: Power and the Production of History. Boston: Beacon.

[56] von Borries, B. (2003), "The Third Reich in German history textbooks since 1945", Journal of Contemporary History, 38(1), 45-62. 
[57] Zerubavel, Y. (1995), Recovered Roots: Collective Memory and the Making of Israeli National Tradition (Chicago, IL). 


\section{Appendix 1}

\section{Proof of Proposition 1}

For all $r \in[q, 1]$ and $g \geqslant 0$, define:

$$
B(r, g)=\frac{1}{2}\left[S W_{S}\left(m_{L}, r\right)-S W_{T}\left(m_{L}\right)\right]=\int_{m_{L}}^{m(r)}\left\{(1+g) m_{L}-c\right\} d F(c)
$$

Lemma 1. For all $r \in[q, 1]$, there exists a unique $G(r)>0$ such that $B(r, G(r))=0$ and:

(i) $B(r, g)>0$ for all $g>G(r)$, while $B(r, g)<0$ for all $g<G(r)$;

(ii) $G(r)<\frac{r\left(m_{H}-m_{L}\right)}{m_{L}}$ and $G(r)$ is strictly increasing in $r$.

Proof of Lemma 1. For any given $r$, it is clear from (8.1) that $B(r, g)>0$ for $g \geqslant \frac{r\left(m_{H}-m_{L}\right)}{m_{L}}$, while $B(r, 0)<0$. Moreover, for all $g \geqslant 0$, we have

$$
\frac{\partial B(r, g)}{\partial g}=\int_{m_{L}}^{m(r)} m_{L} d F(c)>0
$$

This establishes that there is a unique value $G(r)$ such that $B(r, G(r))=0$, and that $0<G(r)<\frac{r\left(m_{H}-m_{L}\right)}{m_{L}}$. Moreover, it establishes part (i) of Lemma 1. It remains to establish that $G^{\prime}(r)>0$. We have:

$$
\frac{\partial B(r, g)}{\partial r}=\left(m_{H}-m_{L}\right)\left[(1+g) m_{L}-m(r)\right] f(m(r))
$$

Moreover, for all $G(r)$ such that $0<G(r)<\frac{r\left(m_{H}-m_{L}\right)}{m_{L}}$, we have:

$$
m(r)>m_{L}(1+G(r))
$$

implying that $\frac{\partial B(r, g)}{\partial r}<0$. Therefore, by the implicit function theorem, $G^{\prime}(r)>$ 0 .

To prove Proposition 1 using Lemma 1, note that:

(i) for all $g>G(1)$ we have, for all $r \in[q, 1], G(r)<g$, and therefore $B(r, g)>$ 0 . Thus the principal's optimal strategy is $h=0$;

(ii) for all $g<G(q)$ we have, for all $r \in[q, 1], G(r)>g$, and therefore $B(r, g)<0$. Thus the principal's optimal strategy is $h=1$; 
(iii) for $G(q) \leqslant g \leqslant G(1)$, there exists by the lemma a unique inverse function $R(g) \equiv G^{-1}(g)$, such that $B(R(g), g)=0$. Moreover, the function $R$ is increasing, and for any $r \in[q, 1], B(r, g)$ has the sign of $R(g)-r$. This implies that the only equilibrium with $r>R(g)$ is $r=1(h=1)$, with $B(1, g)<0$; the only equilibrium with $r<R(g)$ is $r=q(h=0)$, with $B(q, g)>0$; and finally $r=R(g)$ is an equilibrium, with $h=h(g) \equiv \frac{1-\frac{q}{R(g)}}{1-q}$, and $B(R(g), g)=0$.

To complete the proof, define $g_{H} \equiv G(1)$ and $g_{L} \equiv G(q)$.

\section{Proof of Proposition 2}

From the proof of Proposition 1 we know that when the agents believe that the principal always tells the truth (i.e. $r^{*}=1$ ), the principal's optimal strategy is:

(i) for all $g>g_{H}, h=0$;

(ii) for all $g<g_{L}, h=1$;

(iii) for $g_{L} \leqslant g \leqslant g_{H}, h=1$.

\section{Proof of Proposition 3}

For all $r \in\left[q_{N}, 1\right], r_{B B} \in\left[0, \frac{q_{B}}{q_{N}+q_{B}}\right], r_{B G} \in\left[0, \frac{q_{M}}{q_{N}+q_{M}}\right], r_{G B} \in\left[0, \frac{q_{M}}{q_{N}+q_{M}}\right]$, $V \geqslant 0, b \geqslant 0$ and $g \geqslant 0$, define the net gain from suppressing the signal $s=[G, B]$ :

$$
\begin{aligned}
\Re= & \Re\left(r, r_{B B}, r_{B G}, r_{G B}, V, b, g\right)=S W_{S}\left(m_{H}, m_{L}\right)-S W_{T}\left(m_{H}, m_{L}\right) \\
= & -\int_{m_{i}}^{m_{H}}\left[m_{H}(1+V)-c\right] d c+\int_{m_{L}}^{m_{j}}\left[m_{L}(1+V)-c\right] d c \\
& +\int_{b m_{L}}^{b m_{j}}\left[(b+g) m_{L}-c\right] d c-\int_{b m_{i}}^{b m_{H}}\left[(b+g) m_{H}-c\right] d c
\end{aligned}
$$

where

$$
m_{i}=\frac{q_{N} m_{M}+q_{M}\left(1-h_{B G}^{*}\right) m_{L}+q_{M}\left(1-h_{G B}^{*}\right) m_{H}+q_{B}\left(1-h_{B B}^{*}\right) m_{L}}{q_{N}+q_{M}\left(1-h_{B G}^{*}\right)+q_{M}\left(1-h_{G B}^{*}\right)+q_{B}\left(1-h_{B B}^{*}\right)}
$$

and 


$$
m_{j}=\frac{q_{N} m_{M}+q_{M}\left(1-h_{B G}^{*}\right) m_{H}+q_{M}\left(1-h_{G B}^{*}\right) m_{L}+q_{B}\left(1-h_{B B}^{*}\right) m_{L}}{q_{N}+q_{M}\left(1-h_{B G}^{*}\right)+q_{M}\left(1-h_{G B}^{*}\right)+q_{B}\left(1-h_{B B}^{*}\right)}
$$

Letting $x \equiv m_{j}-m_{L}$ and $y \equiv m_{H}-m_{i}$, some manipulation yields:

$$
\Re=(V+b g)\left(m_{L} x-m_{H} y\right)-\frac{1}{2}\left(1+b^{2}\right)\left(y^{2}+x^{2}\right)
$$

It is straightforward to verify that $x \leqslant y$. Hence $\Re<0$, and the principal never suppresses the signal.

\section{Appendix 2}

This appendix provides some of the details that were left out of the exposition in the main text, and referred to in footnotes (31) and (37).

(i) Section 4.1: the benchmark model

The principal's optimal choice of communication strategy, $h$, given the agents' beliefs $h^{*}$ (or equivalently $r^{*}$ ), is obtained by solving:

$$
h \in \arg \max \left[h S W_{T}\left(m_{L}\right)+(1-h) S W_{S}\left(m_{L}, r^{*}\right)\right]
$$

The first-order condition for this problem (which is necessary and sufficient for the solution) is given by:

$$
\begin{array}{cc}
S W_{T}\left(m_{L}\right)-S W_{S}\left(m_{L}, r^{*}\right) \geqslant 0 ; & h=1 \\
S W_{T}\left(m_{L}\right)-S W_{S}\left(m_{L}, r^{*}\right) \leqslant 0 ; & h=0 \\
S W_{T}\left(m_{L}\right)-S W_{S}\left(m_{L}, r^{*}\right)=0 ; & 0<h<1
\end{array}
$$

Thus, as might be expected, if the net gain from signal manipulation, equal to $S W_{S}\left(m_{L}, r^{*}\right)-S W_{T}\left(m_{L}\right)$, is strictly positive, the principal will always suppress the bad signal; if the net gain is strictly negative, he will always tell the truth; finally, if the net gain is equal to zero, the principal is indifferent, and $h$ can take any value in the interval $[0,1]$.

I focus on Perfect Bayesian equilibria, which satisfy the following conditions:

$$
h^{*} \in \arg \max \left[h S W_{T}\left(m_{L}\right)+(1-h) S W_{S}\left(m_{L}, r^{*}\right)\right]
$$




$$
r^{*}=\frac{q}{q+(1-q)\left(1-h^{*}\right)}
$$

\section{(iii) Section 5: the model with heterogeneous agents}

I use the following notation for uninformed beliefs: each agent believes that $s=[\emptyset, \emptyset]$ with probability $q_{N}, s=[B, B]$ with probability $q_{B}, s=[B, G]$ with probability $q_{M}$, and $s=[G, B]$ with probability $q_{M}$, where the subscript $N$ stands for "no news", the subscript $B$ for "bad news", and the subscript $M$ for "mixed news". Given our assumptions about preferences, agent $A_{i}$ will invest in his own culture $\left(x_{i i}=1\right)$ if, and only if,

$$
E\left[m_{i} \mid \hat{s}\right] \geqslant c
$$

Moreover, he will invest in the other agent's culture $\left(x_{i j}=1\right)$ if, and only if,

$$
b E\left[m_{j} \mid \hat{s}\right] \geqslant c
$$

The same applies to agent $A_{j}$.

To compute the conditional expected values of $m_{i}$ and $m_{j}$, it is again useful to define the "reliability" of a "no news" signal, which is given by:

$$
\begin{gathered}
r^{*}=\operatorname{Pr}\left[s=[\emptyset, \emptyset] \mid \hat{s}=[\emptyset, \emptyset] ; h_{B B}^{*}, h_{G B}^{*}, h_{B G}^{*}\right] \\
=\frac{q_{N}}{q_{N}+q_{M}\left(1-h_{B G}^{*}\right)+q_{M}\left(1-h_{G B}^{*}\right)+q_{B}\left(1-h_{B B}^{*}\right)}
\end{gathered}
$$

We shall also need the following notation:

$$
\begin{aligned}
& r_{B B}^{*}=\operatorname{Pr}\left[s=[B, B] \mid \hat{s}=[\emptyset, \emptyset] ; h_{B B}^{*}, h_{G B}^{*}, h_{B G}^{*}\right] \\
& r_{B G}^{*}=\operatorname{Pr}\left[s=[B, G] \mid \hat{s}=[\emptyset, \emptyset] ; h_{B B}^{*}, h_{G B}^{*}, h_{B G}^{*}\right] \\
& r_{G B}^{*}=\operatorname{Pr}\left[s=[G, B] \mid \hat{s}=[\emptyset, \emptyset] ; h_{B B}^{*}, h_{G B}^{*}, h_{B G}^{*}\right]
\end{aligned}
$$

where each probability is obtained applying Bayes' rule.

The agents' expected value of $m_{i}$ conditional on each possible signal $\hat{s}$ transmitted by the principal is then given by:

$$
E\left\{m_{i} \mid \hat{s}=[\emptyset, \emptyset]\right\}=r^{*} m_{M}+r_{B B}^{*} m_{L}+r_{B G}^{*} m_{L}+r_{G B}^{*} m_{H} \equiv m_{i}\left(r^{*}\right)
$$




$$
\begin{aligned}
& E\left\{m_{i} \mid \hat{s}=[B, B]\right\}=m_{L} \\
& E\left\{m_{i} \mid \hat{s}=[B, G]\right\}=m_{L} \\
& E\left\{m_{i} \mid \hat{s}=[G, B]\right\}=m_{H}
\end{aligned}
$$

The expected value of $m_{j}$ conditional on each possible signal $\hat{s}$ is similarly given by:

$$
\begin{gathered}
E\left\{m_{j} \mid \hat{s}=[\emptyset, \emptyset]\right\}=r^{*} m_{M}+r_{B B}^{*} m_{L}+r_{B G}^{*} m_{H}+r_{G B}^{*} m_{L} \equiv m_{j}\left(r^{*}\right) \\
E\left\{m_{j} \mid \hat{s}=[B, B]\right\}=m_{L} \\
E\left\{m_{j} \mid \hat{s}=[B, G]\right\}=m_{H} \\
E\left\{m_{j} \mid \hat{s}=[G, B]\right\}=m_{L}
\end{gathered}
$$

